\title{
Low Power Respiration Monitoring using Wearable 3D knitted Helical Coils.
}

\author{
K. Kiener, A. Anand, W. Fobelets and K. Fobelets, Senior Member, IEEE
}

\begin{abstract}
We demonstrate a novel low power inductive wearable plethysmography system. This consists of ultrasensitive 3D knitted helical coils integrated in a garment and an oscillator circuit with high quality factor. The low power oscillator is built using two cross coupled FET pairs with low capacitance drawing only $95 \mu \mathrm{A}$ during operation and with a response time smaller than $10 \mu \mathrm{s}$. The sensor system is linear, with negligible hysteresis. The best compromise in sensitivity and power consumption is obtained with a 3D knitted helical coil using jersey knit with elastic yarn, a lower knitting needle size than recommended for the yarn and minimizing both the number of stitches per winding as well as the stitches containing metal. A sensitivity of $2.7 \mathrm{kHz}$ per $\mathrm{mm}$ change in circumference with a power consumption of $6.85 \mathrm{~mW}$ per $30 \mathrm{~ms}$ measurement
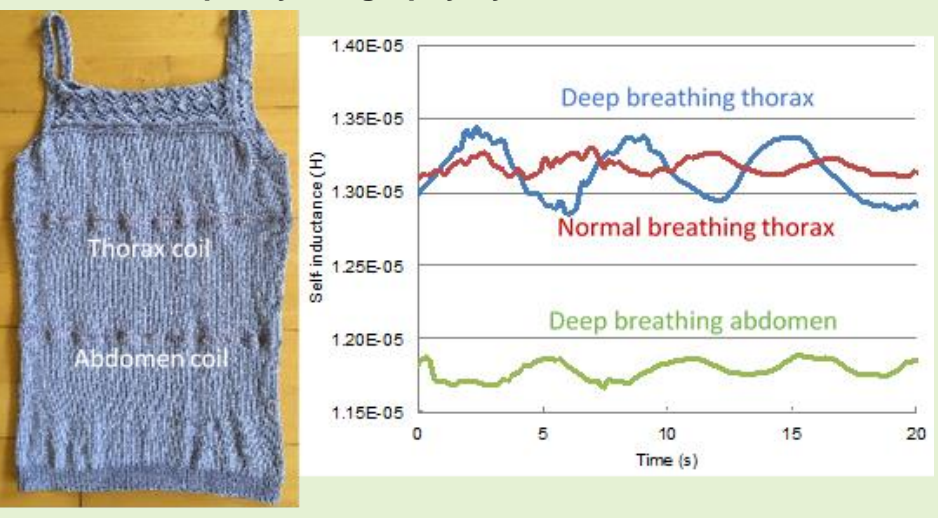
time is reported. This system can be used for long term breathing monitoring using a garment indistinguishable from everyday clothing.
\end{abstract}

Index Terms—MOFET Oscillator, Plethysmography, Wearable Sensor

\section{Introduction}

$\mathrm{M}$ ONITORING respiration signals including respiration rate, breathing depth, phase difference between thorax and abdomen movement and other breathing related parameters, is a useful tool in tracking human health, fitness and wellbeing [1][2]. Respiration monitoring can be used in a multitude of applications including clinical diagnosis and rehabilitation [3][4], sport performance and fitness observation [5] and health and safety support in hazardous occupations [6]. Different sensor approaches exist, broadly put into invasive and non-invasive categories [7]. The first type is normally used in a clinical setting with closely monitored subjects who are willing to undergo this diagnostic approach. The latter, mainly non-clinical approach, is an area extensively researched by the wearable sensors' community [8]. Apart from the sensor performance requirements given by parameters such as linearity, sensitivity, range, accuracy and hysteresis, a wearable sensor needs to fulfil additional needs such as wearing comfort, user acceptance and power consumption of the readout electronics that runs on small, light-weight batteries or energy scavenging systems.

Manuscript received October 15, 2021; revised November 22, 2021; accepted November 23, 2021. The associate editor coordinating the review of this article and approving it for publication was Prof. $\mathrm{Yu}-\mathrm{Te}$ Liao. (Corresponding author: K. Fobelets.)

K. Kiener, A. Anand, and K. Fobelets are with the Department of Electrical and Electronic Engineering, Imperial College London, London SW7 2BT, U.K. (e-mail: k.fobelets@imperial.ac.uk). W. Fobelets is with Barfo, 1750 Lennik, Belgium.

Digital Object Identifier 10.1109/JSEN.2021.3131267.
For wearable breathing monitoring, techniques based on sensing stress or elongation of elastic belts worn around the thorax and/or abdomen, are often used [8]. The sensing mechanisms include resistive, piezoresistive and inductive methods. An overview of commercialized wearable sensors can be found in [9]. Generally, the implementation of wearable inductive plethysmography sensors comes in three forms. One, where the belts are separate from any garment. The second, a strap where the belt is integrated as an elastic band of e.g. a sports bra, and third is a shirt where two belts are integrated within the garment at suitable positions. Looking at inductive plethysmography, we observe that inductive sensing is implemented in all cases using an insulated metal wire integrated in an elastic band with the metal shaped in a sinusoidal or alternative periodic waveform. This conductive wire waveform allows elongation and contraction when the elastic band is stretched and relaxed during breathing. The basic principle of inductive plethysmography is a variation of the self-inductance, $L$ of the belt due to a variation of its circumference during breathing because $L \propto A$, with $A=l^{2} / 4 \pi$ the cross-sectional area and $l$ the circumference.

In this work, we investigate a new implementation of a wearable inductive sensor, first introduced in [10]. It consists of a 3D knitted helical coil formed by circular knitting of both a yarn and a thin insulated metal wire simultaneously. This forms a 3D helical knitted coil that has a behavior like classical wound coils. This sensor implementation is ultimately wearable as it has the knit's natural flexibility and is unobtrusively integrated in a snug fitting garment that 
cannot be distinguished from normal clothing. We use thin $\mathrm{Cu}$ magnet wire with a modified polyurethane resin coating. Polyurethane is the base ingredient in spandex, often used in sportswear. The abrasion resistant coatings show excellent protection against moisture and solvents. The feasibility of using this 3D knitted helical coils for plethysmography was demonstrated in a baby romper [11]. In Fig. 1 circular knitting is demonstrated with 4 knitting needles, together with a closeup of the stitches with yarn and metal wire. A top with a 3D knitted helical coil at thorax and abdomen level demonstrates a possible implementation. The variation of inductance of these coils as a function of breathing of the test subject is shown in the abstract. Those measurements used a Wayne Kerr Precision Component Analyzer 6500B. The resistance of the coil does not change during breathing, but its variation with body temperature can be used to measure body temperature simultaneously [12].

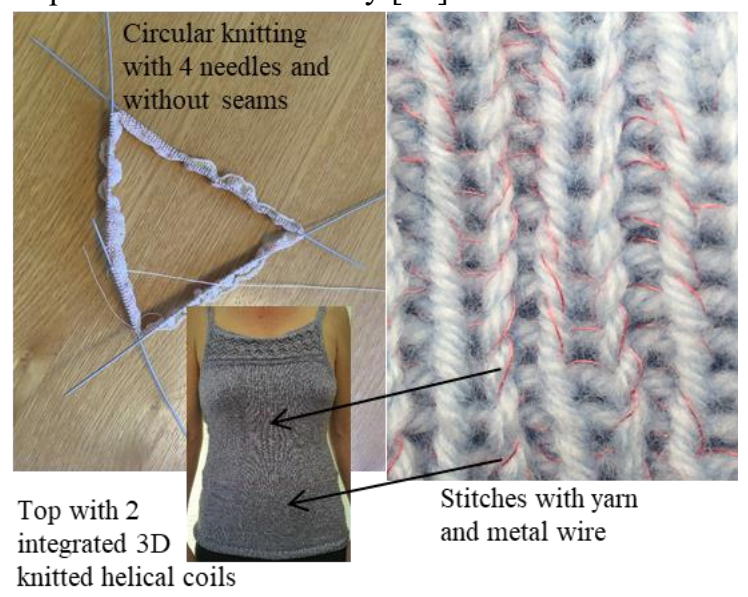

Fig. 1. Pictures of circular manual knitting with 4 needles and 1 yarn, the coil stitches where yarn and metal are knitted together (metal is the thin red wire) and the top with two coils, one integrated at the level of the thorax and one at lower abdomen. The measurements in the inset are done with the test subject wearing this top.

Apart from its wearability and classical look, we show in this work that with careful choice of the knit parameters, the sensor achieves high sensitivity and excellent linearity. We present the study on improving the sensitivity of the knitted coil in terms of yarn, stitch, gauge and number of rows with metal wire (= number of windings, $N$ ) and present a system with a low power readout circuit that boosts the quality factor of the oscillator built around the coil and allows digital readout that conserves power. Low power is achieved by using two cross-coupled MOSFET pairs for the oscillator circuit with low capacitance $\mathrm{n}$ - and p- FETs. A digital counter measures changes in the oscillation frequency with breathing. The counter and oscillator are switched on and off by a microcontroller running at $4 \mathrm{MHz}$ to conserve power. The readout electronics is powered by two flat cell $3 \mathrm{~V}$ batteries.

The manuscript is organized as follows: section II investigates the knit parameters to achieve the best sensitivity of the knitted coil as a breathing sensor. Section III describes the readout electronics using a low power oscillator that increases the quality factor of the circuit and section IV demonstrates the operation of the system. Section V concludes the current work.

\section{Optimisation OF the 3D HELICAL COIL}

To support the measurements, a small chest phantom, shown in Fig. 2, with minimum dimensions of $22 \mathrm{~cm}$ along the long axis and $15.6 \mathrm{~cm}$ along the short axis was $3 \mathrm{D}$ printed. The model of the chest was created using Fusion 360 from Autodesk. The rounded grey and black material represents the ribs, connected with simple hinges [13] to the spine with triangular cross section, in a way that allows them to move in a limited range. The ribs are held together with rubber bands behind the ellipse-shaped sternum (laser cut). For static measurements, plastic plates with a thickness of $0.93 \mathrm{~mm}$ each were inserted or removed between the ribs (grey/black) and the sternum (yellow). Dynamic breathing is implemented by rotating the sternum using a small MicroServo SG90 motor from TowerPro, positioned on top of a plastic rectangular strip connected to the spine. In dynamic measurements an Arduino controls the servo motor to rotate the sternum over specific angles and speed. Investigations show that any potential electromagnetic interference between the servo motor and the coil is negligible.

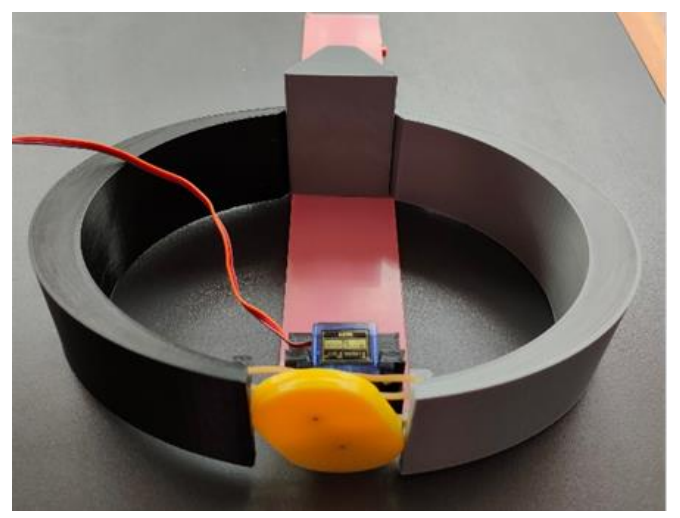

Fig. 2. 3D printed chest phantom with moving ribs connected to a spine and the movement is controlled by a shaped sternum (yellow, laser cut) attached to a servo motor.

The performance of the knitted coil was studied as a function of the number of rows that contain metal wire, $N$; the yarn used (elastic and non-elastic); the stitch type (jersey and $1 / 1$ rib) and the gauge (needle size for a type of yarn). In addition, metal can be integrated in all stitches or only in some, with the metal wire floating at the back of the stitch. We study the impact of having metal wire integrated in all stitches in a row and in every other stitch (denoted with $100 \%$ and $50 \%$ metal, respectively). Its impact is a reduction of the resistance, inductance and elasticity. To limit the impact on elasticity, metal wire is always floated for the same stitch in the wale direction (see Fig. 3) of the knit, allowing full elasticity of the other stitches. This elasticity comes from the horseshoe character of the stitches in the knit. Fig. 3 shows schematic diagrams of the different knit implementations considered in this work.

The performance of the 3D knitted helical coils is given in terms of linearity, sensitivity, resistance and circumference. Linearity is expressed using the coefficient of determination $R^{2}$ and sensitivity as $s=\Delta L / \Delta l(L$ self-inductance, $l$ elongation of the circumference). 
To study the influence of the number of windings $N$, coils were knitted using cotton yarn with needle size $3 \mathrm{~mm}$ and 1/1 rib stitch (1 knit and 1 purl stitch alternated, see Fig. 3) and an insulated $\mathrm{Cu}$ metal wire ( $250 \mu \mathrm{m}$ total thickness) included in all stitches in a row. Measurements were done using a Wayne Kerr 6500B Precision Component Analyzer with an ac signal amplitude of $50 \mathrm{mV}$ at $200 \mathrm{kHz}$, well below the self-resonance frequency of the coils $\left(f_{\text {res }}>20 \mathrm{MHz}\right)$. Thin plastic plates were inserted between sternum and ribs of the phantom to increase the circumference statically in small steps as described before. The variation of $L$ with $l$ is linear with an $R^{2}$ factor larger than 0.99 in all cases. The sensitivity, $s$ as a function of $N$ is given in Fig. 4. $s$ increases approximately proportionally with $N^{2}$.

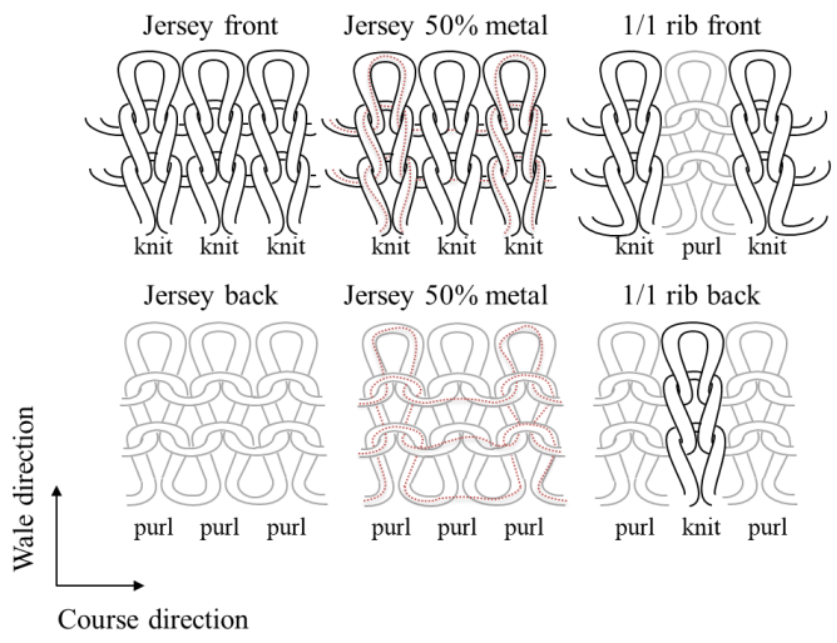

Fig. 3. Schematic drawings of the stitch patterns used in this work illustrating the difference between jersey and rib stitch and the implementation of $50 \%$ metal. Black denotes knit stitches, grey purl stitches and dashed red lines the metal wire. At the back in jersey $50 \%$, the floating metal wire is clearly visible. $100 \%$ metal is when the metal is implemented in all stitches (not drawn).

While this could conclude that the higher $N$ the better the sensor, care needs to be taken concerning the linear increase of the coil's resistance with $N$. Which would hence have an impact on the quality factor of the coil around which the oscillator for the readout circuit is built.

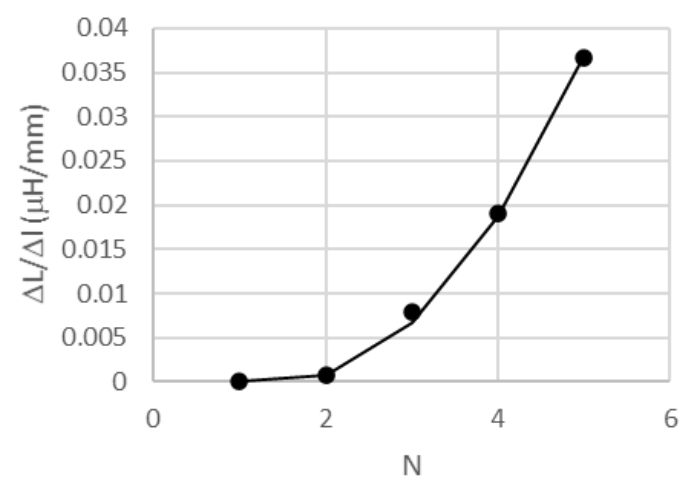

Fig. 4. Sensitivity, $\mathrm{s}$ of the coil as a function of number of windings, $\mathrm{N}$. Cotton yarn is used with $3 \mathrm{~mm}$ needles in 1/1 rib stitch and metal is included in all stitches in the coil. Circles: extracted from measurements. Full line: $\mathrm{N}^{2}$ fit.

The resistance of the coil in $1 / 1$ rib cotton with $3 \mathrm{~mm}$ needles with 128 stitches per row in function of number of windings is given by: $R=2.9 N+0.015(\Omega)$. The constant of $15 \mathrm{~m} \Omega$ is the access resistance of the wire to the coil. In addition, the length of the coil in the garment is limited by the length of the torso of the wearer thus restricting $N . s=57$ $\mathrm{nH} / \mathrm{mm}$ for $N=9$ in this implementation, a value that is much higher than that of $s=1 \mathrm{nH} / \mathrm{mm}$ given in [14] for a typical inductive belt implementation based on an undulating metal wire sewn in an elastic strap.

While cotton is in general well tolerated by most people, elastic yarn, based on acrylic can give additional elasticity to the knit, allowing tighter knits that are still classical in feel and shape. The $N=9$ knit for cotton is repeated in elastic acrylic yarn with different variations in knit parameters.

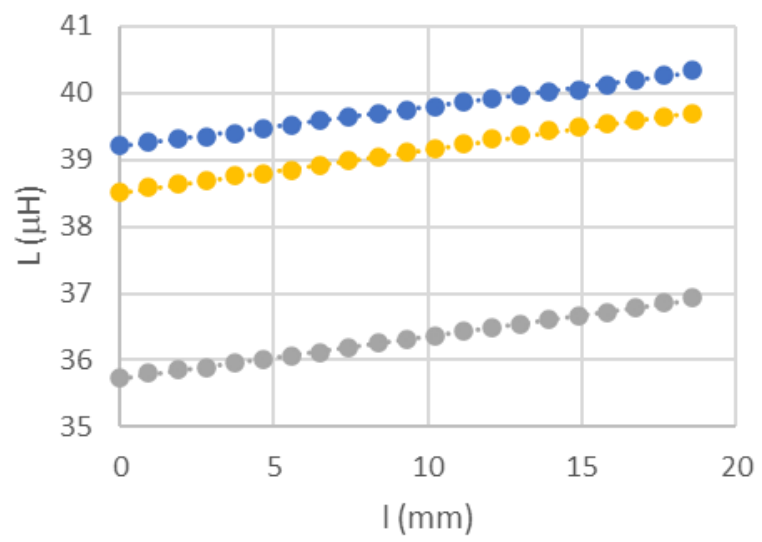

Fig. 5. Self-inductance, $L$ as a function of elongation, $I$ of the circumference for $N=9$. The yarn used is elastic acrylic, needles $3 \mathrm{~mm}$ and 1/1 rib (blue) or jersey (yellow, grey). Grey: metal wire only in $50 \%$ of the stitches. Dashed lines are linear fits through the measurements.

Fig. 5 shows the variation of $L$ as a function of $l$ in $1 / 1 \mathrm{rib}$ (same implementation as in the cotton example in Fig. 4), in jersey and with $100 \%$ and $50 \%$ of metal in the knit (see Fig. 3 ). The number of stitches in the knit is kept constant at 128 . The linearity of $L$ as a function of $l$ remains high with $R^{2}>$ 0.99 for all knits. $1 / 1$ rib stitch gives the highest value of inductance compared to jersey mainly due to the difference of the wire crossings between different stitches in the wale direction, as observed before [15]. The 50\% metal in the stitches gives a lower inductance due to the larger average separation between the windings, however, also has the lowest resistance. The resistance of the coils is $R=24.6,23.8,14.6 \Omega$ for $1 / 1 \mathrm{rib}(100 \%$ metal), jersey (100\% metal) and jersey $(50 \%$ metal), respectively. To compare, the resistance of the cotton rib implementation (Fig. 4) with $N=9$ is $26.3 \Omega$, higher than the implementation in elastic acrylic yarn. The extracted characteristics for the coils are given in Table I and show that $s$ is slightly higher for elastic yarn than cotton and is higher for jersey than $1 / 1 \mathrm{rib}$. The latter confirms results in [11].

In Table I, the characteristics of additional knits with the same number of stitches (128), elastic yarn and stitch type (jersey) but with different needle size are also given. Changing needle size changes knitting gauge and thus total length and width of the coil. This has an impact on the inductance, resistance, sensitivity and circumference of the coil. Decreasing needle size whilst keeping the number of stitches the same (128), 
increases the sensitivity and does not impact on the linearity $R^{2}>0.99$. The circumference of the knitted coil also changes when needle size changes, especially when increasing the size of the needles. Thus, for thicker knitting needles the circumference of the knitted coil at rest increases and thus the fit on the body is looser, influencing sensitivity.

TABLE I

SENSITIVITY S, REST CIRCUMFERENCE Lo, AND RESISTANCE R FOR ELASTIC ACRYLIC YARN AS A FUNCTION OF GAUGE (NEEDLE SIZE), STITCH TYPE AND $\%$ STITCHES WITH METAL. 128 STITCHES/ROW ARE USED.

\begin{tabular}{|l|l|l|l|l|l|}
\hline $\begin{array}{l}\text { needle } \\
\text { size } \\
(\mathrm{mm})\end{array}$ & $\begin{array}{l}\text { stitch } \\
\text { type }\end{array}$ & $\begin{array}{l}\% \text { metal } \\
\text { stitches/row }\end{array}$ & $\begin{array}{l}S \\
(\mathrm{nH} / \mathrm{mm})\end{array}$ & $l_{0}(\mathrm{~cm})$ & $R(\Omega)$ \\
\hline 3 & $1 / 1$ rib & 100 & 59.5 & $41 \pm 0.3$ & 24.6 \\
\hline 3 & jersey & 100 & 64.8 & $44.4 \pm 0.3$ & 23.8 \\
\hline 3 & jersey & 50 & 63.5 & $43.3 \pm 0.3$ & 14.6 \\
\hline 3.5 & jersey & 100 & 59.1 & $47.8 \pm 0.3$ & 27 \\
\hline 2.5 & jersey & 100 & 63 & $44.2 \pm 0.3$ & 22.5 \\
\hline 2.5 & jersey & 50 & 68.5 & $44.2 \pm 0.3$ & 14 \\
\hline
\end{tabular}

Decreasing the needle size also decreases the resistance as the total length of metal/yarn used is smaller since the length of the coil is smaller. A low coil series resistance is important as it improves the quality factor of the coil and thus will impact on the operation of the electronic readout circuit. Therefore, coils were also knitted with metal in $50 \%$ of the stitches only. This is done by knitting a stitch with yarn and metal followed by a stitch with yarn only with the metal passing behind the work to the next stitch that is knitted with yarn and metal again (see Fig. 3). This is repeated, creating alternate metal/no metal stitches. This is a technique as used in jacquard knitting [16].

TABLE ॥

SENSITIVITY S, REST CIRCUMFERENCE Lo, AND RESISTANCE R FOR ELASTIC ACRYLIC YARN AS A FUNCTION OF GAUGE (NEEDLE SIZE), STITCH TYPE AND $\%$ STITCHES WITH METAL. 138 STITCHES/ROW ARE USED

\begin{tabular}{|l|l|l|l|l|l|}
\hline $\begin{array}{l}\text { needle } \\
\text { size } \\
(\mathrm{mm})\end{array}$ & stitch type & $\begin{array}{l}\text { \% metal } \\
\text { stitches/row }\end{array}$ & $\begin{array}{l}\mathrm{s} \\
(\mathrm{nH} / \mathrm{mm})\end{array}$ & $l_{0}(\mathrm{~cm})$ & $\begin{array}{l}R \\
(\Omega)\end{array}$ \\
\hline 2.5 & jersey & 50 & 61 & $46 \pm 0.3$ & 14.3 \\
\hline 3.5 & jersey & 50 & 63 & $48 \pm 0.3$ & 18.2 \\
\hline 3.5 & jersey & 100 & 61 & $52 \pm 0.3$ & 29.3 \\
\hline 3.5 & jersey twist & 100 & 68 & $52 \pm 0.3$ & 30.2 \\
\hline
\end{tabular}

The metal wire pulled behind the stitches without incorporated metal decreases the elasticity of the coil. To limit loss in elasticity, the stitches with metal and no metal overlap in the wale direction of the knit. From Table I, the resistance with $50 \%$ metal is much lower. Also, the inductance is lower (see Fig. 5). Concerning the sensitivity, these measurements do not give a conclusive answer but seem to indicate that with lower needle size and 50\% metal, the sensitivity increases.

To give more insight, further studies were done using a higher number of stitches per row, equal to 138 , with different needle sizes. A jersey knit with a twisted stitch in alternate stitches in the course direction is added. A twist stitch is made by putting the needle in the back rather than the front of the loop. This twists the bottom of the stitch influencing the inductance of the coil as the metal wire overlap changes. Results are given in Table II.

The sensitivity of the coil is dependent on the stitch type, its circumference at rest, and its elasticity, influenced by the presence of floating metal stitches. An insight into the best knit for coil sensitivity can be extracted from Fig. 6 where the sensitivity of all coils is plotted as a function of rest circumference.

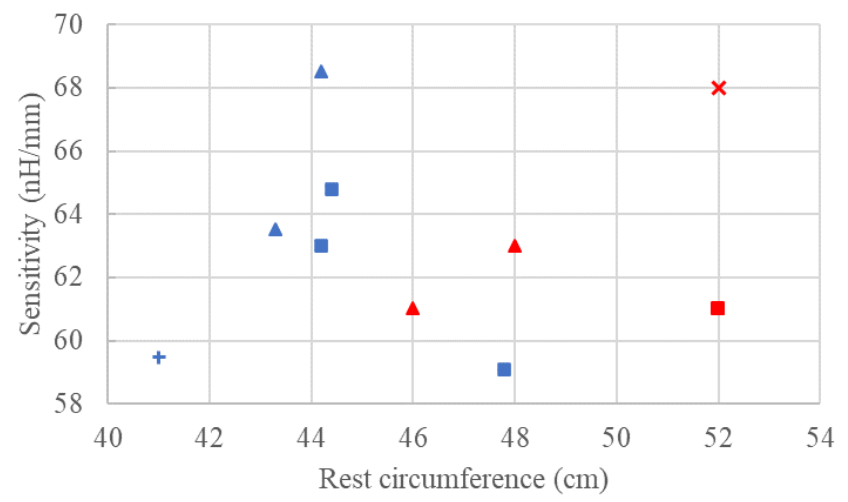

Fig. 6. Sensitivity as a function of rest circumference of the knitted coil. 128 and 138 stitches are blue and red, respectively. 100\% metal: squares, $50 \%$ metal: triangles. + is $1 / 1$ rib and $x$ is jersey twist.

Fig. 6 shows a trend that indicates that smaller diameters give higher sensitivity. 50\% metal implementations boost the sensitivity, interesting in view of their lower resistance. $1 / 1$ rib shows the lowest sensitivity and the jersey twist the highest. This variation in response shows that the way in which the metal wires overlap between neighboring stitches in the wale direction has an important influence. Stretching the coil not only changes its diameter but also forces the legs of the stitches to reposition [17] causing changes in inductance [18].

\section{LOW POWER READOUT ELECTRONICS}

To read the variations of the self-inductance during breathing electronically, a resonator is built around the coil. This converts the inductance changes with circumference into frequency changes. The oscillator frequency change is read by a digital counter controlled by a microcontroller [19]. In this section we present a low power readout circuit that operates on two $3 \mathrm{~V}$ flat cell batteries. 

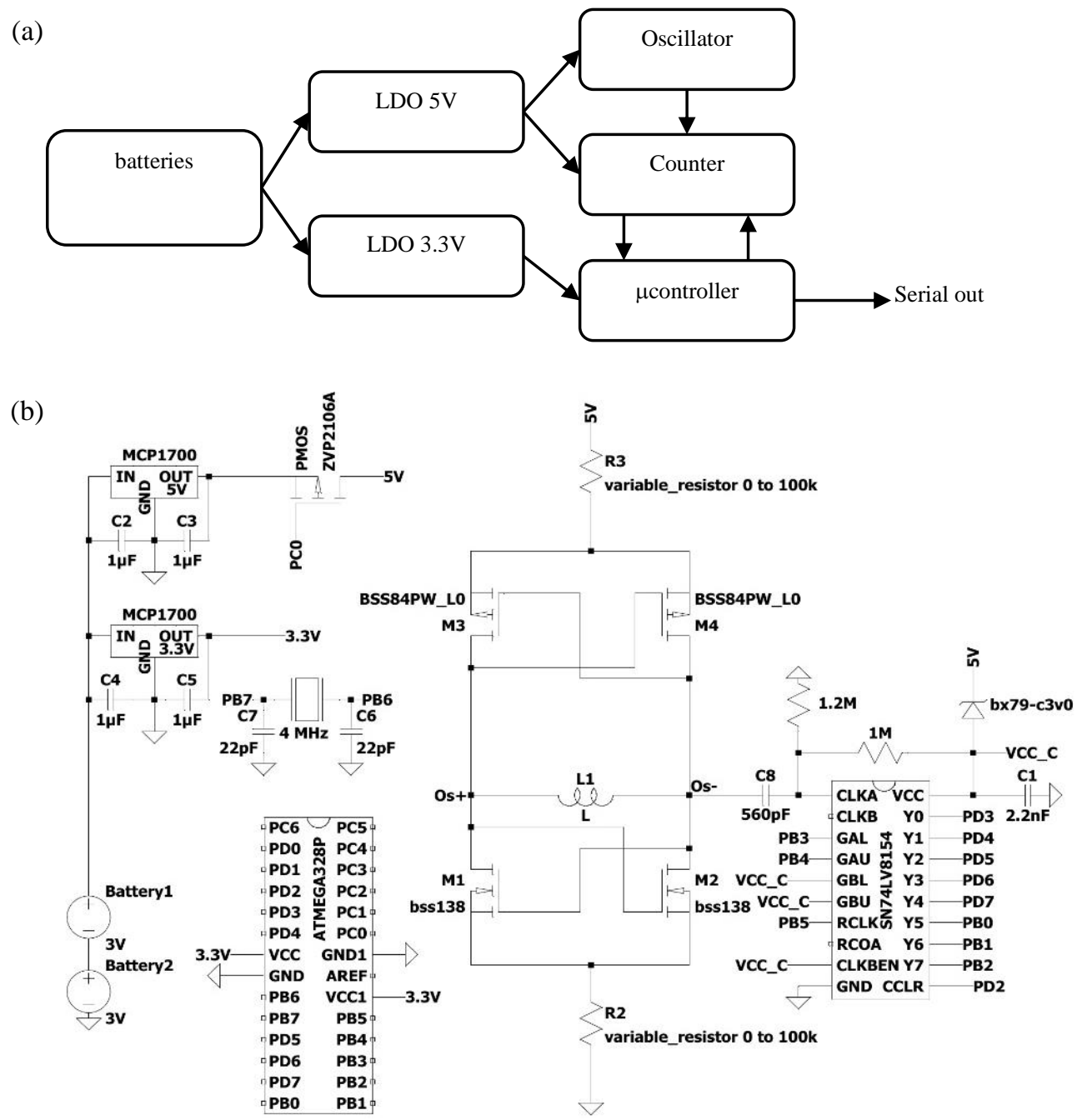

Fig.7. (a) Block diagram of the readout circuit powered by two flat cell batteries of $3 \mathrm{~V}$ each. (b) The circuit diagram.

The variable inductance represents the coil.

Fig. 7 shows the low power readout circuit for the knitted coil. The heart of the electronic readout is the oscillator circuit based on two cross-coupled pairs [20]. A SM74LU8154 dual 16-bit binary counter is used to translate inductance to frequency. An ATMEGA328P microcontroller clocked by an external $4 \mathrm{MHz}$ crystal oscillator controls the counter and the ZVP2106A pMOS that switches the system on and off to conserve power.

In the oscillator, both cross coupled FETs pairs form a negative resistance circuit with a resistance given by:

$$
R^{-1}=\frac{-g_{m}}{2}\left(1-\frac{1}{g_{m} \times R_{0}}\right)
$$

$g_{m}$ is the transconductance and $R_{0}$ the output resistance of the FETs that are assumed to be the same.

The top cross coupled pair of pFETs makes connection to the power supply, ensures $g_{m} \times R_{o}>1$ - the oscillation condition in eq. (1) - and together with the nFET determines the capacitors for the LC tank circuit (with $L$ the variable coil inductor and $C$ the capacitance of $\mathrm{C}_{\mathrm{pFET}} / / \mathrm{C}_{\mathrm{nFET}}$ ). The oscillator's frequency is given by:

$$
f=\frac{1}{2 \pi \sqrt{L C}}
$$

Low capacitance nFETs and pFETs are used to boost the quality factor, $Q$ of the LC tank circuit and compensate for the negative impact of the high coil resistance. The quality factor $Q$ is given by:

$$
Q=\frac{1}{R} \sqrt{\frac{L}{C}}
$$

A low capacitor also increases the frequency shift due to the variation of the coil inductance:

$$
\Delta f=f_{0+\Delta}-f_{0}=\frac{1}{2 \pi \sqrt{C}}\left(\frac{1}{\sqrt{L_{0}+\Delta L}}-\frac{1}{\sqrt{L_{0}}}\right)
$$

With $f_{0}$ and $L_{0}$ the oscillator frequency and self-inductance of the coil at rest and $\Delta$ the variations in value due to circumference changes (breathing). This equation indicates that coils with lower inductance will give higher $\Delta f$.

In this implementation, R2 and R3 (Fig. 7) are variable resistors that allow the characterization of the wide range of knitted coils studied in this work. Once their value is determined for a specific coil $(N$ and $R)$ and choice of FETs, they can remain constant. The resistors control the current and thus power and set the amplitude $\Delta V$ and offset $V_{D C}$ of the oscillating voltage. This is important for the counter in the next stage to work correctly. In addition, they compensate for the variation of the threshold voltage between the different discrete FETs and are responsible for keeping the FETs in the triode region to decrease power wasted in harmonics and 
harmonics interfering with the surroundings. To prevent the battery's voltage drop over time to influence the circuit performance, a low-voltage drop-out regulator (LDO) is used that gives a constant $5 \mathrm{~V}$ output to the oscillator. The MCP1700 was chosen for its small quiescent current: $\sim 1.6 \mu \mathrm{A}$. The LDO output voltage only varied by $0.004 \mathrm{~V}$ for a battery voltage change of $1 \mathrm{~V} .1 \mu \mathrm{F}$ capacitances are added to remove the noise generated by the LDO.

The SN74LV8154 digital frequency counter is directly connected to the oscillator. To reduce power consumption, we only use one counter with 8 bits. Its $\mathrm{V}_{\mathrm{CC}}$ voltage should be in the range of $2 \mathrm{~V}$ to $5.5 \mathrm{~V}$. A low $\mathrm{V}_{\mathrm{CC}}$ voltage was chosen to reduce the current consumption of the counter. The oscillator input signal to the counter is tuned to go from nearly GND to $\mathrm{V}_{\mathrm{CC}}$ to reduce power consumption of the internal Schmitt trigger. To supply $\mathrm{V}_{\mathrm{CC}}$ to the counter, we use a reverse biased Zener diode, defined at $3 \mathrm{~V}$ but delivering $2.6 \mathrm{~V}$ in the experimental set-up. The voltage divider ensures the diode works in Zener mode, supplying $\sim 80 \mu \mathrm{A}$. The $560 \mathrm{pF}$ capacitor $\mathrm{C} 8$ removes the offset from the oscillator signal. The minimum input voltage of $700 \mathrm{mV}$ from the oscillator for the counter to work is set by R2 and R3.

The ATMEGA328P microcontroller was chosen to read out the counter. Its drawback is its high power consumption. Different approaches were taken to minimise its power consumption whilst maintaining measurement integrity.

First, an external $4 \mathrm{MHz}$ clock is chosen to reduce the ATMEGA328P power consumption while still maintaining a high processing rate and an accurate clock [21]. This $4 \mathrm{MHz}$ crystal oscillator is connected to two $22 \mathrm{pF}$ ceramic capacitors as shown in fig. 7.

Second, the power supply to the microcontroller is reduced to $3.3 \mathrm{~V}$ using a $2^{\text {nd }} \mathrm{LDO}$.

Third, breathing is sampled during $\sim 15 \mathrm{~ms}$ only ( $5 \mathrm{~ms}$ setup time and $10 \mathrm{~ms}$ counting time). The system is active for $\sim 45$ $\mathrm{ms}$ and is in sleep mode for $250 \mathrm{~ms}$. The microprocessor's watchdog timer controls the pMOS that switches the counter and oscillator on and off. Only $7 \mu \mathrm{A}$ is drawn by the microcontroller in deep sleep mode during the $250 \mathrm{~ms}$ with the watchdog on. The LDO also stays on in sleep mode but draws only $1.6 \mu \mathrm{A}$ current. Upon on-switching, the oscillator stabilizes within $10-20 \mu \mathrm{s}$, much shorter than the counter reset time of $1-2 \mathrm{~ms}$.

In the current implementation, the microcontroller sends the information at the end of the measurement via a serial port to a host computer.

Fig. 8 compares the variations of the inductance measured by the Wayne Kerr Precision Component Analyzer with that from the electronic readout. This demonstrates a reasonably accurate measurement of the variation of the coil's inductance. The measurement frequency is converted into an inductance by assuming the FET capacitance of the LC tank remains constant at $40.8 \mathrm{pF}$. The amplitude difference is mainly due to the values of the capacitance of the tank resonator that is determined by the exact DC operation point of the FETs.

The symmetry in the upward and downward slopes in the measurements in Fig. 8 show negligible hysteresis in the coil's and system's performance.

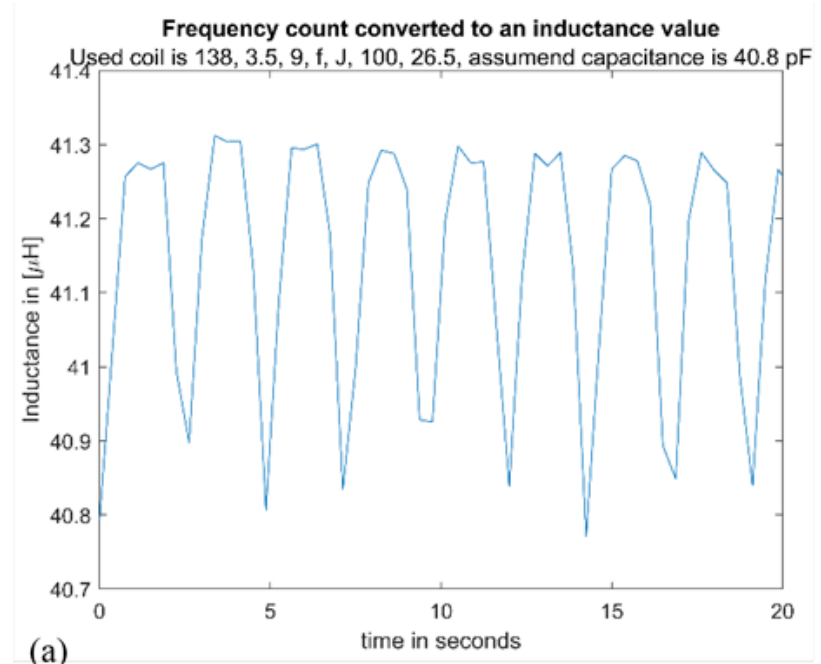

(a)

Inductance read-out with precision analyser Wayne Kerr $6500 \mathrm{~B}$

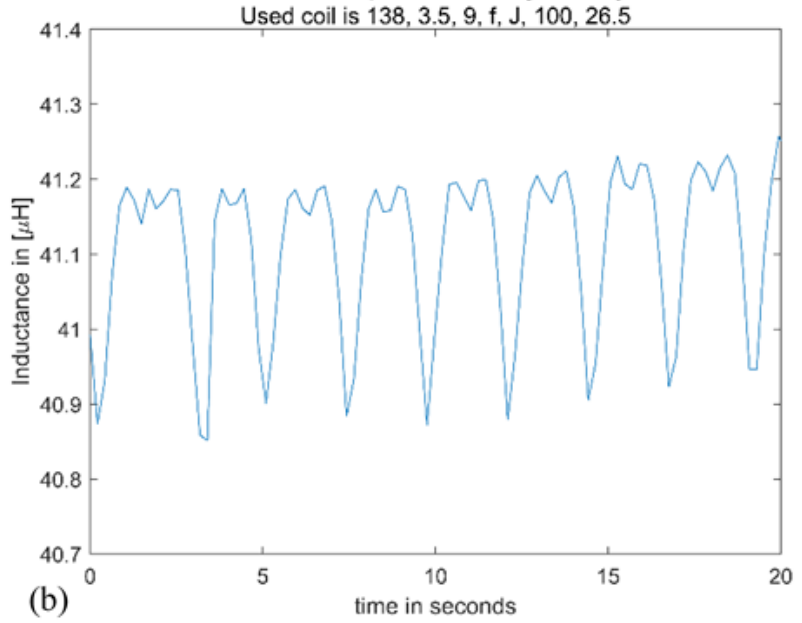

Fig. 8. Simulated breathing signal using the phantom and rotating the sternum with the motor over $\pm 60^{\circ}$, a) read by the circuit, b) using the Wayne Kerr precision component analyzer. The component analyzer has more measurement points. The double peak at the top is related to the shape of the moving sternum. The knitted coil has 128 stitches, a $3.5 \mathrm{~mm}$ needle, 9 turns, jersey knitting technique, $100 \%$ metal and elastic yarn.

\section{AnAlysis of PERformance}

Section II showed that the sensitivity of the knitted coils can be optimized via different knit parameters (type of yarn, stitch type, diameter of coil, \%stitches with metal per winding). A high number of windings and a tighter knit in jersey stitch tend to give higher coil sensitivity. In the following, we analyze the performance of the system for the different knitted coils. We look at linearity, sensitivity and power consumption. The latter is important as the coil needs to be worn for long periods of time without the need for battery recharging.

We repeat the sensitivity measurements using the readout circuit. We find linear variations of frequency with changing circumference for all coils over the circumference range measured, $R^{2}>0.9$. The performance of the oscillator is summarised in Table III. The resistors R2 and R3 are changed to keep the peak-to-peak and offset voltage of the oscillations the same for all coils. The frequency of coils with lower $N$ is higher because of eq. (2). In the last two columns, the frequency sensitivity to circumference changes is given. The 
one denoted by "Wayne Kerr" is derived from the static frequency measurements with the precision component analyzer and $C=84 \mathrm{pF}$, estimated from the $N=9$ measurement. The last column is derived from the circuit measurements. The larger difference between the two values for lower $N$ is due to the deviation of $C=84 \mathrm{pF}$ due to differences in bias conditions of the FETs as a function of $N$. Both approaches show decreasing sensitivity to circumference variations for increasing $N$.

While these results indicate a preference for coils with a lower number of windings (consistent with eq. (3)), column 2 shows that the current drawn by the circuit is lower for a higher number of windings and thus is preferable for longer battery lifetime. Increasing $N$ decreases the current drawn from the batteries because the quality factor of the coil increases as a function of $N^{0.3}[18]$.

TABLE III

THE CURRENT, PEAK-TO-PEAK VOLTAGE, OFFSET VOLTAGE, OSCILLATION FREQUENCY $F_{0}$ WITH THE COIL AT REST. THE VALUES OF THE RESISTORS R2 AND R3 TO TUNE THE PEAK-TO-PEAK AND OFFSET VOLTAGES TO BE AROUND $1 \mathrm{~V}$. THE VARIATION OF THE FREQUENCY WITH INCREASING CIRCUMFERENCE FROM REST. THE KNITTED COILS ARE THE COTTON 1/1 RIB SAMPLES WITH NEEDLE SIZE 3 MM, 128 STITCHES AND METAL IN ALL

\begin{tabular}{|c|c|c|c|c|c|c|c|c|}
\hline$z$ & 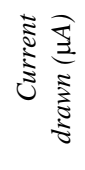 & 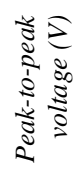 & 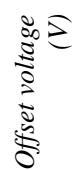 & 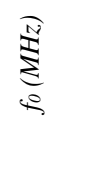 & 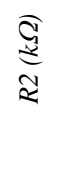 & $\begin{array}{l}\frac{\widehat{c}}{2} \\
\frac{1}{2} \\
\stackrel{2}{2}\end{array}$ & 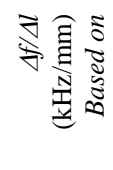 & 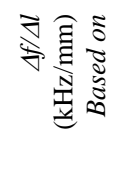 \\
\hline 9 & 95 & 1.05 & 1.07 & 2.75 & 9.6 & 26.2 & -1.9 & -1.9 \\
\hline 7 & 114 & 1.01 & 1.07 & 3.35 & 8.6 & 22.2 & -2.3 & -2.1 \\
\hline 5 & 168 & 1.03 & 0.96 & 4.27 & 4.6 & 15 & -2.4 & -3.5 \\
\hline 3 & 266 & 0.98 & 1.06 & 6.2 & 3.3 & 9.5 & -3.0 & -4.1 \\
\hline 1 & 883 & 1.09 & 1 & 13.32 & 1.1 & 3 & -2.5 & -5.3 \\
\hline
\end{tabular}

Microcontroller and counter draw additional current from the battery. The total power consumption can be calculated using:

$$
P=\frac{V_{D D}}{T} \int_{0}^{T} i_{D D}(t) d t
$$

with $\mathrm{V}_{\mathrm{DD}}$ the battery voltage, assumed to remain constant. $i_{D D}=V_{100} / R$ with $R=100 \Omega$, the resistance across which the $V_{100}$ voltage drop is measured. A plot of $i_{D D}$ as a function of time for the 1/1 rib $N=9$ cotton coil is given in Fig. 9a. The regions where the current is low is when the microcontroller is in sleep mode. The other regions indicated with numbers is when the microcontroller is awake. (1) is when the controller switches the pMOS on, resets the counter and starts the oscillator. During (2) the counter measures the oscillation frequency. In (3) the microcontroller reads out counter and in (4) the microcontroller transmits the data. Data transmission consumes the largest amount of power.

The results of eq. (4), ignoring region (4) in Fig. 9a, are given in Fig. 9b for the 1/1 rib implementations in cotton as a function of number of windings. Region (4) is ignored as this is the same for all coils. This demonstrates that the power consumption is decreasing with an increasing number of windings.

Taking $N=9$, thus prioritizing power consumption, for the knits with elastic yarn in jersey stitch and looking at the influence of other knit parameters, we find that the power consumption is lowest for the $50 \%$ metal implementations (see Fig. 10).
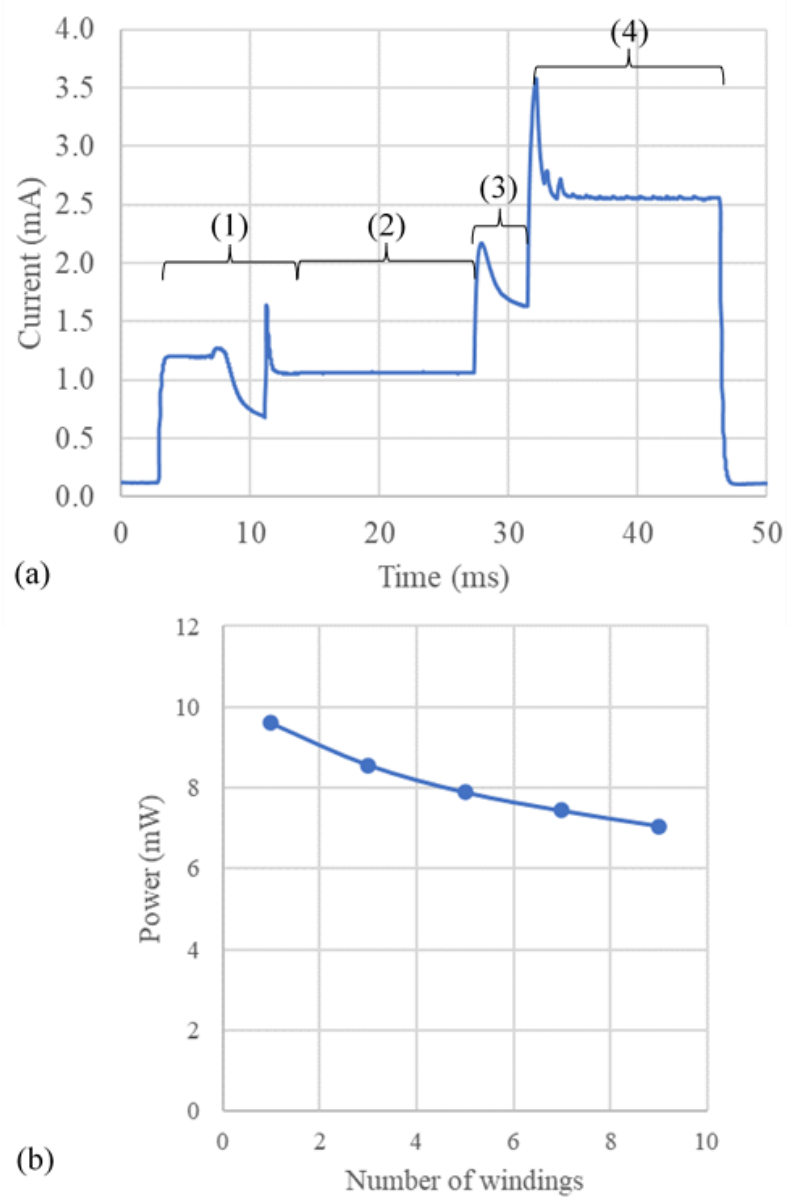

Fig. 9. (a) Current drawn by the system when $\mathrm{ON}$ for the $1 / 1$ rib $\mathrm{N}=9$ cotton coil (100\% metal and $3 \mathrm{~mm}$ needles). (1) reset of counter, start oscillator and wait, (2) counter measures oscillation frequency, (3) microcontroller reads out counter, (4) microcontroller transmit info. Elsewhere, system in deep sleep. (b) Power consumption during awake time of the microcontroller. The power consumed in region (4) is not considered.

As for the system sensitivity $(\Delta f / \Delta l)$ we find that a jersey knit with elastic yarn, small needle size $(2.5 \mathrm{~mm})$ and $50 \%$ metal wire per winding, outperforms the other implementations. Thus, power consumption can be reduced by both increasing the number of windings (increasing coil quality factor) and decreasing the number of stitches with metal (reducing the resistance of the coil). The sensitivity can then be improved by using a jersey knit with a smaller needle size than prescribed for the yarn used (higher gauge, tighter knit).

With the chosen microcontroller, the power used to transmit the information to the auxiliary via serial cable, tends to overshadow the gains made in coil optimization, calling for a less power hungry microcontroller. The power consumed during deep sleep is orders of magnitude smaller than that reported in Fig. 10 because the leakage current is of the order of $10 \mu \mathrm{A}$, a factor of 100 smaller than the current drawn when the system is $\mathrm{ON}$. 


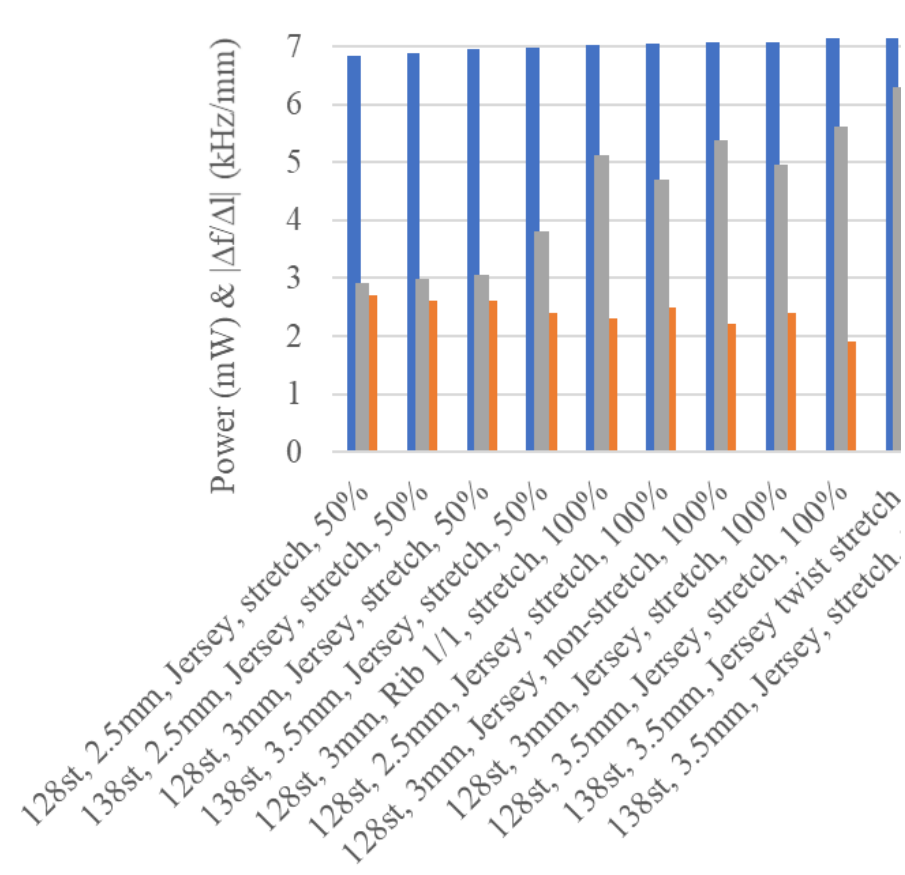

Fig. 10. The power (blue), absolute value of the frequency sensitivity $|\Delta \mathrm{f} / \Delta| \mid$ (orange) and resistance (grey) for different knits with $N=9$.

These measurements were carried out with a chest phantom that only changes its circumference. This neglects noise superimposed by movement of the wearer. In those more complex situations multiparameter sensing e.g. simultaneous measurement of breathing, ECG and gyroscope movement sensing together with signal processing techniques will need to be implemented for denoising the signal.

\section{CONCLUSION}

3D knitted helical coils show large sensitivity to changes in circumference. Their sensitivity is up to 68 times higher than that of a classical sinusoidal coil integrated in a strap. All coils show linear changes in inductance with circumference with negligible hysteresis. A higher number of windings and a tighter knit in elastic yarn, using jersey stitch gives the best coil sensitivity.

The inductance change is converted to a frequency change using a low power oscillator based on two cross coupled FET pairs. The low capacitance FETs boost the quality factor of the oscillator making it low power with fast response time. The variation of the frequency with circumference is linear with negligible hysteresis. A frequency change of $5.3 \mathrm{kHz} / \mathrm{mm}$ is obtained for $N=1$ but the lowest power consumption of 6.85 $\mathrm{mW} /$ reading is obtained for $N=9$ (exclusive the data transmission). The power hungry microcontroller is put in deep sleep mode for $250 \mathrm{~ms}$ and the breathing signal is sampled for $15 \mathrm{~ms}$ every $280 \mathrm{~ms}$.

Using a relatively tight fit in jersey with only $50 \%$ of metal in the stitches of each winding gives the best power-sensitivity compromise and still allows easy variations of the circumference with breathing while not hindering its wearability.

\section{REFERENCES}

[1] A. Aliverti, "Wearable technology: role in respiratory health and disease." Breathe 13: e27-e36, 2017.

[2] A. Angelucci, M. Cavicchioli, I.A. Cintorrino, G. Lauricella, C. Rossi, S. Strati, A. Aliverti, "Smart Textiles and Sensorized Garments for Physiological Monitoring: A Review of Available Solutions and Techniques.", Sensors 21, 814, 2021.

[3] S. Patel, H. Park, P. Bonato, L. Chan and M. Rodgers, "A review of wearable sensors and systems with application in rehabilitation." $J$ NeuroEngineering Rehabil 9, 21, 2012.

[4] L.M.S.d. Nascimento, L.V. Bonfati, M.L.B. Freitas, J.J.A. Mendes Junior, H.V. Siqueira, and S.L. Stevan, Jr. "Sensors and Systems for Physical Rehabilitation and Health Monitoring-A Review." Sensors 20, 4063, 2020. [5] S. Scataglini, A.P. Moorhead, and F. Feletti, "A Systematic Review of Smart Clothing in Sports: Possible Applications to Extreme Sports." Muscles Ligaments Tendons J. 10, 333, 2020.

[6] G. Tartare, X. Zeng, and L. Koehl, "Development of a wearable system for monitoring the firefighter's physiological state." In Proceedings of the IEEE Industrial Cyber-Physical Systems (ICPS), St. Petersburg, Russia, 15-18 May 2018; pp. 561-566.

[7] A. S. Ginsburg, J. L. Lenahan, R. Izadnegahdar, and J. M. Ansermino, "A Systematic Review of Tools to Measure Respiratory Rate in Order to Identify Childhood Pneumonia" Am J Respir Crit Care Med 197(9), pp 1116-1127, 2018.

[8] V. Monaco, and C. Stefanini, "Assessing the Tidal Volume through Wearables: A Scoping Review", Sensors 21, 4124, 2021.

[9] S. Jayasekera, E. Hensel and R. Robinson, "Feasibility Assessment of Wearable Respiratory Monitors for Ambulatory Inhalation Topography”, Int. J. Environ. Res. Public Health 18, 2990, 2021.

[10] K. Fobelets, K. Thielemans, A. Mathivanan, and C. Papavassiliou, "Characterization of Knitted Coils for e-Textiles", IEEE Sensors J. 19(18), pp. 7835-7840, 2019.

[11] K. Fobelets, "Knitted coils as breathing sensors", Sensors and Actuators A: Physical 306, 111945, 2020.

[12] K. Fobelets, Ambulatory monitoring using knitted 3D helical coils, $3^{\text {rd }}$ Int. Conf. Challenges, Opportunities, Innovations and Applications in Electronic Textiles, 3-4 Nov 2021 Manchester, UK.

[13] Quick Hinge by solertron - Thingiverse (www.thiniverse.com/thing:47591). Accessed on 1 Feb 2021.

[14] H.T. Ngo, C.V. Nguyen, T.M.H. Nguyen, and T.V. Vo, "A Portable Respiratory Monitor Using Respiratory Inductive Plethysmography", IFMBE proceedings Jan 2013, 40. Pp. 222-225 In book: 4th International Conference on Biomedical Engineering in Vietnam (pp.222-225) Chapter: 57 Publisher: Springer Berlin Heidelberg.

[15] K. Fobelets "Knitted coil for inductive plethysmography", Proceedings 2019, 32, 2. (in International Conference on the Challenges, Opportunities, Innovations and Applications in Electronic Textiles).

[16] E. Wood, "Formation and Properties of Knitted Structures.", The Australian Wool Education Trust (2009) Microsoft Word - Wool-482-582-08T-20.docx (woolwise.com).

[17] S. Poincloux, M. Adda-Bedia, and F. Lechenault, "Geometry and Elasticity of a Knitted Fabric.", Phys. Rev. X 8, 021075, 2018.

[18] K. Fobelets, K. Sareen, and K. Thielemans, "Magnetic coupling with 3D knitted helical coils", in press Sensors and Actuators: A. Physical (online 11 Nov. 2021). https://doi.org/10.1016/j.sna.2021.113213

[19] K. Kiener, W. Fobelets, and K. Fobelets, "Respiratory Inductive Plethysmography System using Knitted 3D Helical Coils." $3^{\text {rd }}$ Int. Conf. Challenges, Opportunities, Innovations and Applications in Electronic Textiles, 3-4 Nov 2021 Manchester, UK.

[20] M. Ebrahimzadeh, "Design of an ultra-low power low phase noise CMOS LC oscillator.", International Journal of Soft Computing and Engineering (IJSCE), 1(4) pp. 78-81, 2011.

[21] Atmel / Microchip, "8-bit avr microcontroller with 32k bytes in-system programmable flash datasheet," 2015. [Online]. Available: https://ww1.microchip.com/downloads/en/ DeviceDoc/Atmel-7810Automotive-Microcontrollers-ATmega328P Datasheet.pdf 